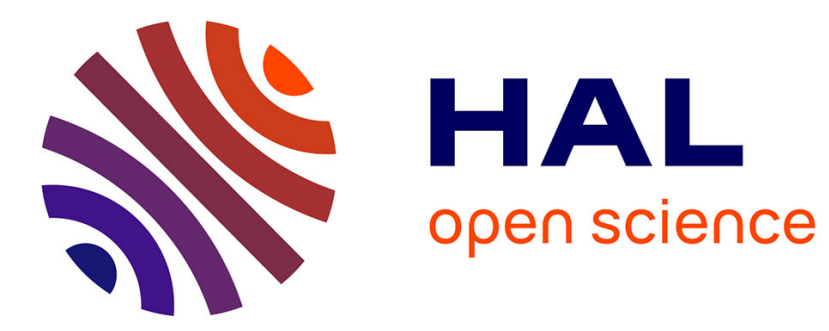

\title{
Interstitial gas effect on vibrated granular columns
}

Javier C. Pastenes, Jean-Christophe Géminard, Francisco Melo

\section{To cite this version:}

Javier C. Pastenes, Jean-Christophe Géminard, Francisco Melo. Interstitial gas effect on vibrated granular columns. 2014. hal-00999226

\section{HAL Id: hal-00999226 \\ https://hal.science/hal-00999226}

Preprint submitted on 4 Jun 2014

HAL is a multi-disciplinary open access archive for the deposit and dissemination of scientific research documents, whether they are published or not. The documents may come from teaching and research institutions in France or abroad, or from public or private research centers.
L'archive ouverte pluridisciplinaire HAL, est destinée au dépôt et à la diffusion de documents scientifiques de niveau recherche, publiés ou non, émanant des établissements d'enseignement et de recherche français ou étrangers, des laboratoires publics ou privés. 


\title{
Interstitial gas effect on vibrated granular columns
}

\author{
Javier C. Pastenes ${ }^{\dagger}$, Jean-Christophe Géminard ${ }^{\ddagger}$, and Francisco Melo ${ }^{\star}$ \\ $\dagger$ Departamento de Física Universidad de Santiago de Chile, \\ Avenida Ecuador 3493, 9170124 Estación Central, Santiago, Chile. \\ $\ddagger$ Laboratoire de Physique, Ecole Normale Supérieure de Lyon, \\ Université de Lyon, CNRS, UMR 5672, 46 Allée d'Italie, F-69007 Lyon, France.
}

(Dated: June 4, 2014)

\begin{abstract}
Vibrated granular materials have been intensively used to investigate particle segregation, convection and heaping. We report on the behavior of a column of heavy grains bouncing on an oscillating solid surface. Measurements indicate that, for weak effects of the interstitial gas, the temporal variations of the pressure at the base of the column are satisfactorily described by considering that the column, in spite of the observed dilation, behaves like a porous solid. In addition, direct observation of the column dynamics shows that the grains of the upper and lower surfaces are in free fall in the gravitational field and that the dilation is due to a small delay between their takeoff times.
\end{abstract}

PACS numbers: 45.70.Mg, 45.70.Qj, 81.20.Ev.

\section{INTRODUCTION}

The rapid compression of a relatively loose pile of sand ${ }^{50}$ or of snow may require a high pressure to drive the ${ }^{51}$ flow of the interstitial fluid between the solid particles, ${ }^{52}$ grains or flakes. The effect, together with the elastic ${ }^{53}$ and frictional resistance, contributes to the pressure to ${ }^{54}$ overcome to compress the material. Interestingly, due ${ }^{55}$ to this drainage effect, snowboarding and sandboarding ${ }^{56}$ benefit from a significant lift force and therefore from a ${ }^{57}$ significant reduction of the friction at large slip velocity ${ }^{58}$ if the medium is loose enough [1]. Indeed, viscous forces ${ }^{59}$ are prone to be at play when a gas is evacuated through 60 a wide variety of porous materials frequently found in common life and industrial applications 2]. From physical viewpoint the influence of interstitial viscous forces on non-cohesive granular materials has generated long- 61 lasting debate due mainly to the difficulties introduced 62 by the complex rheology of unconsolidated porous media, 63 and by the sensibility of the response to the conditions 64 imposed at the boundary surfaces. Booming sand [3, [4] 65 and the jets resulting from the impact of a solid object 66 onto the surface of a loosely packed granular bed [5 7] 67 are subtle manifestations of the coupling of the mechan- 68 ical response of granular matter with the dynamics of 69 the interstitial fluid. Heaping, granular convection and 70 size segregation under vibration [8 10] are a few other 71 examples of phenomena in which the internal viscous 72 forces drive, at least partially, the motion of the grains 73 and, thus likely, changes in the external shape of the sys- 74 tem [11, 12].

In the same way, in thin layers of non-cohesive powders 76 submitted to repeated pats, granular droplets appear as 77 a result of the interplay between the air flow through the 78 material, which leads the droplets to grow, and the sta- 79 bility of the granular slopes, which limits their size [13]. 80

\footnotetext{
* francisco.melo@usach.cl
}

In a previous work, we reported on the formation and on the, even more striking, upward motion of millimetric droplets on an incline subjected to vertical vibration [14]. We later showed that the viscous drag, which is of the order of the droplet weight, is responsible for the droplet formation while the gas pressure at the droplet base provides an effective horizontal acceleration whose cumulative effect is an upward displacement of the center of mass after each cycle of the vibration [15]. Interestingly, the experiments revealed that the droplets move only if the maximum acceleration of the substrate is larger than a threshold which we associated, in a first qualitative approach, to a characteristic dilation.

In the present report, we focus on the gas pressure and dilation in a simplified geometry, i.e. a cylindrical granular column subjected to vertical vibration. We limit the study to the regime of low viscous friction by using particles of relatively large size and low frequency of vibration. The main aim of the study is to provide insight into the mechanisms that lead the column to dilate. First, we show that a classical Darcy's law accounts for the dynamics of the gas pressure at a column base. Interestingly, the agreement of our measurements with early predictions obtained by assuming a rigid porous medium [16, 17], indicates that, for sufficiently tall columns, the porosity changes associated with the column dilation have negligible effects. However, even in this limit, a significant overall dilation of the column is observed. From the additional detailed analysis of the system dynamics, we conclude that the granular column not only does not dilate along its whole height but also that, indeed, the dilation only involves the grains of the lower and upper surfaces, which experience slightly delayed free falls. Our results provide a more quantitative way to assess the dilation effects and the role they play in the instabilities observed in related systems, such as those mentioned hereinabove. 


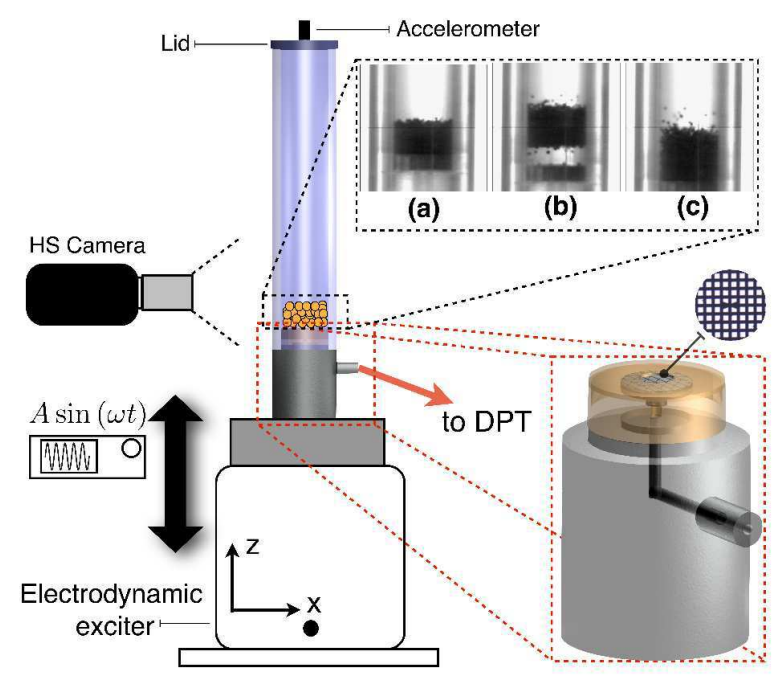

FIG. 1. (Color online) Sketch of the experimental device ${ }^{12}$ The grains inside lye inside a cylindrical container vibrated ${ }^{127}$ vertically. The resulting pressure variations in the gap be- ${ }^{128}$ tween the substrate and the bottom surface of the column, ${ }^{129}$ $\Delta P$, is monitored by means of a differential pressure transducer (DPT) while a high-speed camera is used to observe the dynamics of the column from the side. Bottom-right in-130 set: Details of the L-shaped tube connecting the gap to the DPT and of the grid at the surface of the mount. Top-right inset: Typical images from the camera (a) Initial contact be- ${ }^{131}$ tween the column and the substrate, previous to take-off (b) Large gap underneath the column in flight (c) Sudden land-132 ing of the column [Steel grains, $d=745 \mu \mathrm{m}, h_{0}=5.7 \mathrm{~mm}{ }_{133}$ $f=15 \mathrm{~Hz}$ and $\Gamma=2.6]$.

\section{EXPERIMENTAL SETUP AND PROTOCOL ${ }_{13}$} $z_{1}$

\section{, el}

shorter than $1 \mathrm{~ms}$. Thus, the configuration achieves measurement of the pressure difference, $\Delta P$, with an accuracy of about $2 \mathrm{~Pa}$ in the range $\pm 124 \mathrm{~Pa}$.

The whole is vibrated vertically using an electrodynamic exciter (Labworks, MT-160) fed with a sinusoidal current of frequency, $f$, in the range 15 to $50 \mathrm{~Hz}$. The acceleration of the container, $\gamma(t)$, is monitored by means of a charge accelerometer, placed at the top, its axis aligned with the vertical. From the signal, $\gamma(t)$, we determine, to within 0.01 , the dimensionless acceleration $\Gamma \equiv \max (\gamma) / g=A \omega^{2} / g$, where $A$ stands for the amplitude of the vibration and $g$ for the magnitude of the acceleration due to gravity $(\omega \equiv 2 \pi f)$. In the present study, $\Gamma$ is chosen within the range from 1 to 4 .

The dynamics of the granular material is observed from the side by means of High Speed (HS) video camera. The resolution of the images is of $256 \times 256 \mathrm{px}^{2}$ together with an acquisition rate of $1200 \mathrm{fps}$. The heights, $z_{0}$ and $z_{1}$, of the free surface and of the bottom of the column, respectively, are obtained with a resolution of $0.2 \mathrm{~mm}$ by elementary image analysis.

\section{EXPERIMENTAL RESULTS}

\section{A. General description}

For given vibration frequency $\mathrm{f}$ and dimensionless acceleration $\Gamma$, we report on the dynamics of the granular column and on the temporal evolution of the pressure $\Delta P$ in the steady state (Fig. 22).

First, the dynamics of the column is mainly characterized by the vertical positions, $z_{0}(t)$ and $z_{1}(t)$, of its upper and lower surfaces (Fig. 2a). One observes that, on the one hand, the column periodically looses contact with the substrate, which is better illustrated by displaying the gap, $s(t) \equiv z_{1}(t)-z(t)$, i.e. the vertical size of the region free of grains between the substrate and the column (Fig. 2b). On the other hand, the column periodically dilates, which is clearly revealed by reporting the column height, $h(t) \equiv z_{0}(t)-z_{1}(t)$ (Fig. 2r). The signal from the accelerometer exhibits a significant noise after the gap has vanished until the dilated column recovers its initial height (Fig. 22d) A complex temporal evolution of the pressure $\Delta P(t)$ results from the dynamics of the grains (Fig. 22).

In next section IIIB we interpret qualitatively the behavior of the system. In section IIIC, we discuss thoroughly the temporal behavior of the pressure signal, $\Delta P(t)$, whereas section $\amalg \mathrm{D}$ is devoted to the dynamics of the granular column.

\section{B. Qualitative understanding}

Let us first assume that the column sits at rest on the substrate and that the pressure inside is in equilibrium 

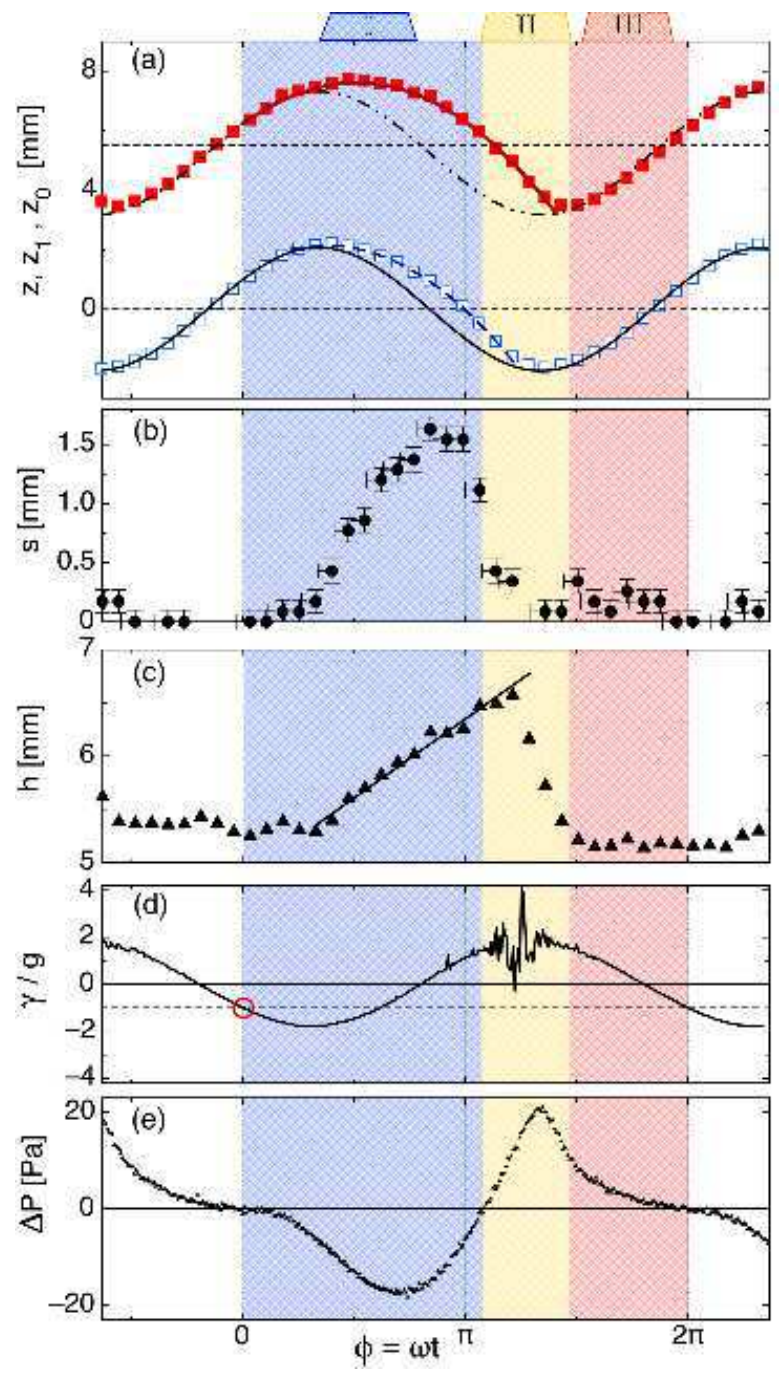

FIG. 2. (Color online) Evolution of column characteristics ${ }_{194}$ and of the pressure as a function of phase $\omega t-(\mathrm{a})$ Vertical $_{195}$ positions of the substrate $z$ (continuous line), of the upper surface $z_{0}$ (full squares) and of the lower surface $z_{1}$ (open squares) vs. phase $\omega t$. Dashed-dotted line: $h_{0}+z$ is a guide for the ${ }^{197}$ eye. Red (light gray) thick line and Blue (dark gray) dashed ${ }^{198}$ line: free fall of the upper and lower surface respectively. The ${ }^{199}$ parabolas have curvature -g. (b) Gap $s(t) \equiv z_{1}(t)-z(t)-200$ In region I (blue), the column is not in contact with the sub-201 strate. (c) Column height $h(t) \equiv z_{0}(t)-z 1(t)-$ The column exhibits a significant dilation in regions I (blue) and II (yellow). Straight line: linear increase of $h$. (d) Acceleration $_{202}$ $\gamma(t)$ - The significant noisy vibration in region II (yellow) is due to the collapse of the column onto the substrate. Red (light gray) circle: $\gamma=-1$. (e) Pressure $\Delta P-$ In region $\mathrm{I}^{203}$ (blue), while the column takes off and dilates, $\Delta P$ decreases, ${ }^{204}$ reaches a minimum and increases again. In region II (yellow),205 $\Delta P$ continues to increase while the column, in contact with206 the substrate, settles back. In a last phase, in region III (red),207 $\Delta P$ decreases while the column seats at rest on the substrate. 208 $\left[h_{0}=5.7 \mathrm{~mm}, f=15 \mathrm{~Hz}\right.$ and $\left.\Gamma=1.81\right]$.

with the outer pressure. Provided that the typical veloc-212

$$
\text { le }
$$
meability of the column, which leads to a relaxation of $\Delta P$ toward the equilibrium with the outside pressure. In our experimental conditions, the observation of a minimum in $\Delta P$ reveals that the characteristic relaxation time, $\tau_{r}$ is of the order of the flight duration (itself of the order of $1 / f$ in the reported example).

\section{Sudden landing}

Due to its fall in the gravity field and to the vertical vibration of the container, the lower surface of the column enters again in contact with the substrate. The system enters region II in Fig. 2. The height $h(t)$ of the column then rapidly recovers its initial value $h_{0}$ (Fig. 2r). This collapse of the column produces the noise seen in the signal from the accelerometer (Fig. 2 d). Provided that the pressure relaxation time, $\tau_{r}$, associated with the gas transport in the column, is larger than the typical collision time, $\tau_{c}$, the pressure, $\Delta P$, still increases as long as the height of the column decreases (Fig. 22 d). As a 
consequence, the maximum of $\Delta P$ is not reached at the 259 collision time but later on, close to the end of the column 260 collapse.

Finally, after the collapse, the column sits at rest on the substrate. The system enters the region III in Fig. $2^{264}$ However, the pressure of the gas in the column is initially ${ }^{265}$ larger than the outer pressure. It relaxes continuously,266 with a characteristic time $\tau_{r}$, toward the outside pres-267 sure because of the resulting gas flow through the grains ${ }^{268}$ (Fig. 22) until the next take-off (Sec. IIIB 1).

\section{Pressure pattern, $\Delta P(t)$}

Here, we introduce a theoretical framework to support the qualitative description proposed in Sec. IIIB.

\section{Take-off and flight}

In a first simplified approach, we consider that the col-272 umn moves as a whole and we neglect the dilation and ${ }^{273}$ the possible grain convection. If the inner pressure is ini-274 tially in equilibrium with the outer pressure, the column ${ }^{275}$ takes off when the downward acceleration of the substrate ${ }^{276}$ equals that of the gravity, thus for $\gamma=-g$. The column ${ }^{277}$ is subsequently flying.

In flight, the column is submitted the gravity and to the force associated with $\Delta P$. Denoting $z_{\mathrm{G}}(t)$ the altitude of the column center of mass, $\mathrm{G}$, we write:

$$
\frac{d^{2} z_{\mathrm{G}}}{d t^{2}}=-g+\frac{1}{\rho h_{0}} \Delta P(t) .
$$

This equation explicitly couples the dynamics of the $\mathrm{col}^{282}{ }_{283}^{22}$ umn with the overpressure $\Delta P$. However, note that the ${ }_{284}$ gas pressure alters the dynamics only if $\Delta P$ is of the or ${ }^{285}$ der of $\rho g h_{0}$, the stress applied by the column onto the ${ }_{286}^{265}$ substrate at rest.

Now, in order to account for the pressure variations in- ${ }^{288}$ duced by the column dynamics, we consider that $\Delta P_{289}^{288}$ induces a gas flow through the grains. The instan- ${ }_{290}$ taneous flow-rate is approximately given by a Darcy ${ }_{291}^{290}$ law, $q=-(\kappa / \eta) \nabla P$, where $\eta$ is the gas viscosity and $_{292}^{291}$ $\kappa$ the permeability given by the Ergun relation, $\kappa={ }_{203}$ $\psi^{3} d^{2} /\left[150(1-\psi)^{2}\right]$, where $\psi$ is the porosity [18]. As- $^{293}$ suming further that the gas is incompressible, we esti- ${ }_{295}$ mate that the variation of the gap $s(t)$ between the col- $^{295}$ umn and the substrate is only permitted by the gas flow, ${ }^{296}$ which imposes that $d s / d t=q$, with $q=(\kappa / \eta)\left(\Delta P / h_{0}\right)^{297}$ from the Darcy law applied to our configuration. $\mathrm{We}_{299}^{298}$ thus have:

$$
\frac{d \Delta P}{d t}=h_{0} \frac{\eta}{\kappa} \frac{d s}{d t}
$$

Thus, combining the equations governing the motion of the column (Eq. 1) and the pressure variations (Eq. 2) and taking into account that, in absence of dilation, $z_{\mathrm{G}}=$ $h_{0} / 2+s+z$, we write:

$$
\frac{d^{2} \tilde{s}}{d \phi^{2}}+\frac{1}{\tilde{\phi}_{\kappa}} \frac{d \tilde{s}}{d \phi}=\sin \left(\phi+\phi_{0}\right)-\frac{1}{\Gamma},
$$

where $\tilde{s} \equiv s / A, \phi \equiv \omega t$ and $\phi_{0} \equiv \omega t_{0}=\arcsin 1 / \Gamma, t_{0}$ being the time of the take-off [i.e. $\gamma\left(t_{0}\right)=-g$ ]. The parameter $\tilde{\phi}_{\kappa} \equiv \omega \kappa \rho / \eta$ is a relaxation time expressed in units of the vibration period. Eq. (3) was first obtained by Kroll for a porous oscillating piston in his pioneering works 16 and it is referred to as the Kroll's model. Eq. (3) has an analytic solution which is written [10]:

$$
\begin{aligned}
\Delta P(\phi) & =-\frac{\rho g h_{0}}{1+\tilde{\phi}_{\kappa}^{2}}\left[\sqrt{\Gamma^{2}-1}\left(\sin \phi-\tilde{\phi}_{\kappa} \cos \phi\right)\right. \\
& +\tilde{\phi}_{\kappa} \sin \phi-\tilde{\phi}_{\kappa}^{2}+\cos \phi \\
& \left.+\tilde{\phi}_{\kappa}\left(\sqrt{\Gamma^{2}-1}+\tilde{\phi}_{\kappa}\right) e^{-\frac{\phi}{\phi_{\kappa}}}-1\right] .
\end{aligned}
$$

The relaxation time $\tilde{\phi}_{\kappa}$ is the characteristic time needed by the column to reach the regime governed by the air viscosity. For small fluid viscosity $\eta$, large density $\rho$ of the material the grains are made of, or large grain diameter $d$ (the porosity scales like $d^{2}$ ), the effect of air is tiny and this time can be large in comparison with the period of the vibration. In the limit $\tilde{\phi_{\kappa}} \gg 1$, the pressure difference $\Delta P(\phi)$ in Eq. (44) exhibits the minimum:

$$
\frac{\Delta P_{\min }}{\rho g h_{0}}=-\frac{1}{\tilde{\phi}_{\kappa}}\left[\arccos \left(\frac{2}{\Gamma^{2}}-1\right)-2 \sqrt{\Gamma^{2}-1}\right]
$$

Interestingly, $\Delta P_{\min }$ depends on one single adjustable parameter, $\tilde{\phi}_{\kappa}$, provided that the acceleration $\Gamma$ and the weight $\rho g h_{0}$ (per unit area) of the column are known.

In Fig. 3a, we report $\Delta P_{\min } /\left(\rho_{s} g h_{0}\right)$ as a function of $\Gamma$ for various column height $h_{0}$ (As the porosity $\psi$ and, thus the density of the column $\rho=(1-\psi) \rho_{s}$, are a priori unknown, we normalized the data using the density of steel $\rho_{s}$ ). First, we observe an excellent collapse of the data on a master curve, except for the thinnest column at large acceleration $\left(h_{0}=2.1 \mathrm{~mm}\right.$ and $\left.\Gamma>2.5\right)$. When the column is too thin and the acceleration too large, the grains do not bounce as a whole but rather form a gaseous phase and, then, the model fails in describing the pressure pattern, $\Delta P(t)$. Except for the thinnest column, the interpolation of the experimental data with Eq. (5) leads to $\tilde{\phi}_{\kappa}=(14.6 \pm 0.1)$ and, thus, to $\psi \simeq 0.51$ (we consider the viscosity of air $\eta=18.610^{-6} \mathrm{~Pa} \mathrm{~s}$ ). The porosity is found to be greater than the porosity of a random loose packing, which is acceptable for a column flying almost freely, not compacted by gravity. The dependence on frequency of $\Delta P_{\min }$ at constant $\Gamma$ constitutes an additional clue that the model is acceptable (Fig. 3b). Note finally 

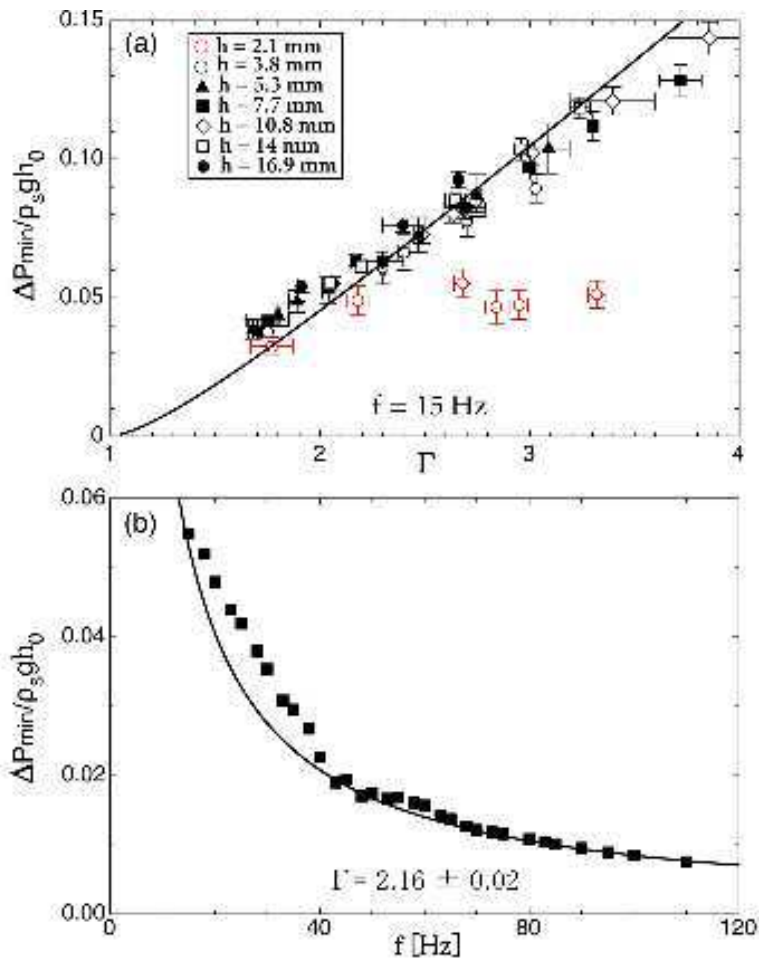

FIG. 3. (Color online) Normalized minimum gap-pressure, ${ }_{349}$ $\Delta P_{\min } /\left(\rho_{s} g h_{0}\right):($ a) dependence on acceleration $\Gamma$ at constant frequency $f=15 \mathrm{~Hz}$. (b) dependence on frequency at constant acceleration $\Gamma=2.16 \mathrm{~Hz}$. Solid line: fit from Eq. (5) with $\tilde{\phi}_{\kappa}=14.6 \pm 0.1$, which leads to $\psi \simeq 0.51$.

that the model remains valid even if the characteristic 354 (normalized) time $\tilde{\phi}_{\kappa}$ is not much larger than the unity. ${ }^{355}$ Nevertheless, the rather large value of $\tilde{\phi}_{\kappa}$ indicates that ${ }^{356}$ the viscosity almost does not alter the trajectory of the $e^{357}$ column that should nearly experience a free flight. The $\mathrm{T}^{358}$ assumption will be discussed in Sec. IIID.

\section{Layer at rest}

After the column-substrate collision, the column col- ${ }_{36}$ lapses and then sits at rest on the solid surface, the in- ${ }_{366}$ ner pressure being initially larger than the outer pressure ${ }_{367}$ (Fig. 2, left of region III). We observe that $\Delta P$ slowly $_{368}$ relaxes towards 0 . However, our crude model cannot ac- ${ }_{369}$ count for this relaxation as $\Delta P$ is expected to vanish vio $_{370}$ when the column moves with the substrate (Darcy law, 371 Sec. IIIC 1). We previously assumed that the compress- ${ }_{372}$ ibility of the gas could be neglected when the grains are $_{373}$ in motion (Sec. III C 1), but we must take it into account ${ }_{374}$ to describe the relaxation of $\Delta P$ when the column is $a_{375}$ rest.

Considering the Darcy law and the adiabatic pressure 377 variation due to the associated gas flow in a granular 378 column of porosity $\psi$, we write the diffusion coefficient ${ }_{379}$ $D=\alpha P_{0} \kappa /[\eta(1-\psi)]$, where $P_{0}$ stands for the outside 380 pressure and $\alpha=1.4$ for the adiabatic constant for dry air. The typical relaxation time in a column of height $h_{0}$ is $\tau=h_{0}^{2} / D$. In our experimental conditions, taking $\psi=0.58$ for the column sitting at rest on the substrate, we estimate $D \simeq 3 \mathrm{~m}^{2} / \mathrm{s}$. For $h_{0}=5.7 \mathrm{~mm}$, we thus get $\tau \sim 10 \mu \mathrm{s}$, much shorter than the time observed experimentally.

In order to recover the experimental relaxation time, one must take into account that the column sits above a pressurized cavity and that the relaxation time is rather due to the escape of the gas trapped underneath. We estimate that the total volume of the L-shaped pipe drilled in the tube mount and of the hose connecting the latter to the pressure transducer, $v_{\text {conn }} \sim 2 \mathrm{~cm}^{3}$. Assuming that the gas escapes only through a cylinder of length $h_{0}$ and radius $r_{p}$ within the column, we expect the resulting characteristic time $\tau=\eta h_{0} v_{\text {conn. }} /\left(\pi r_{p}^{2} \alpha P_{0} \kappa\right)$ to be about $30 \mathrm{~ms}$ for $h_{0}=5.7 \mathrm{~mm}$. This estimate is of the order of the typical relaxation time, of about $5 \mathrm{~ms}$, which is observed experimentally (Fig. 2a). Assuming that the gas escapes only through a tube of radius $r_{p}$ obviously leads to an overestimate but the agreement validates the proposed mechanism of relaxation.

\section{Discussion of the pressure pattern}

We have seen that the pressure pattern is reasonably described by considering two different regimes. In region I, after take-off, the decrease of the pressure, $\Delta P$, and its minimum are recovered by using a Darcy law, while neglecting the compressibility of the gas and the dilation of the column. In region III, the relaxation of the pressure requires the compressibility of the gas to be considered.

In this framework, the evolution of $\Delta P$ while the column settles back onto the substrate (Fig. 2] region II) would require to take both the dilation of the column and the compressibility of the gas into consideration. We mention here that, in this regime, a horizontal front separates a column of grains sitting at rest on the substrate from the grains above that are still in motion. The description proposed in Sec. IIC 1 should remain valid when applied to the grains in motion. This argument at least explains the continuity of the pressure evolution when the column hits the substrate. Indeed, there is no discontinuity of the velocity at the beginning of the contact. In addition, after the contact, the height of the column of grains that are still in motion decreases which explains that the contribution of the grain motion to the pressure variation $d \Delta P / d t$ (Eq.2) decreases. At the same time, the pressure relaxes towards the outer pressure as explained previously in Sec. III C2. As a result of the two effects, the pressure reaches a maximum somewhere in the region II (Fig. 2), before the column completely collapsed and remains sitting at rest on the substrate. At this stage we compare the pressure pattern to former works by Gutman [17]. Indeed, Gutman extended 
the simplified Kroll's model to account for the gas compressibility upon the gas flow through a porous layer and performed pressure measurements beneath the vibrated layer. Although Gutman did not consider the possibility of layer dilation on his model, the calculated pattern contains the main features we observed experimentally (compare Fig. 2 to Fig. 2 in Ref. [17]). The main feature attributed to compressibility effects is that the decay of the air pressure in the column after the collision takes a finite time, so that when the column takes off in the next cycle the gas pressure in the opening gap is above atmospheric. The effect is not significant in our experimental conditions [19].

Finally, we point out that the measurements of $\Delta P$ during the take-off, and direct measurements of the subsequent flight time, indicate that the trajectory of the column is not different from that of a porous solid (for $\Gamma<3)[20,21]$. One can thus wonder how it is then possible to understand that this result is compatible with the observation of a significant dilation. The question will be answered in the next section, in which we even propose a dilation mechanism.

\section{Layer Dilation}

In Fig. 2r, one observes that the column dilates dur- ${ }^{441}$ ing its flight (region I). The dilation of the column can be accounted for, by considering that the behavior of the grains at the upper and lower surfaces differs qual-442 itatively from that of the grains in the bulk of the column. Indeed, at the surface, the grains, in addition $\mathrm{to}_{443}$ the mechanical solid contact with their neighbors below ${ }_{444}$ and above, are submitted to gravity and to the friction ${ }_{445}$ with air which is small and, negligible in our experimen- ${ }_{446}$ tal conditions.

Consider the grains of the first layer at the top of the ${ }_{448}$ column. We observe experimentally that they experience ${ }_{449}$ a free fall, $z_{0}(t)$ (Fig. 2 2 ). To account for this observation ${ }_{450}$ we note that the friction of air has negligible effect on iso- ${ }_{451}$ lated grains or, at least, an effect much smaller than that ${ }_{452}$ on a dense column. As a result, at $\gamma=-1$, the grains $_{453}$ of the free surface take off and detach from the dense ${ }_{454}$ column below whose trajectory, governed by Eq. (4), is 455 always below that expected for a free fall. As a conse- ${ }_{450}$ quence, $z_{0}=A \sin \left(\omega t_{0}\right)+A \omega \cos \left(\omega t_{0}\right)\left(t-t_{0}\right)-\frac{1}{2} g\left(t-{ }_{457}\right.$ $\left.t_{0}\right)^{2}$ where, we remind, $t_{0}$ is the time at take-off.

Interestingly, we observe in Fig. 2r that $h$ increases lin-459 early with time $t$. The height $h$ being defined as the 460 difference between the altitude $z_{0}$ of the upper and $z_{1461}$ lower surfaces, we conclude that the grains at the bot-462 tom also experience a parabolic flight with the same 463 acceleration, thus a free fall. This conclusion is sup-464 ported by the direct observation of the free fall in Fig. $2 \mathrm{a}, 465$ where both (upper and lower) parabolas have curvature 466 -g. The observed linear increase of $h$ with time thus re-467 sults from the fact that the free falls of the grains at ${ }_{468}$ the upper and lower surfaces do not have the same ini-469

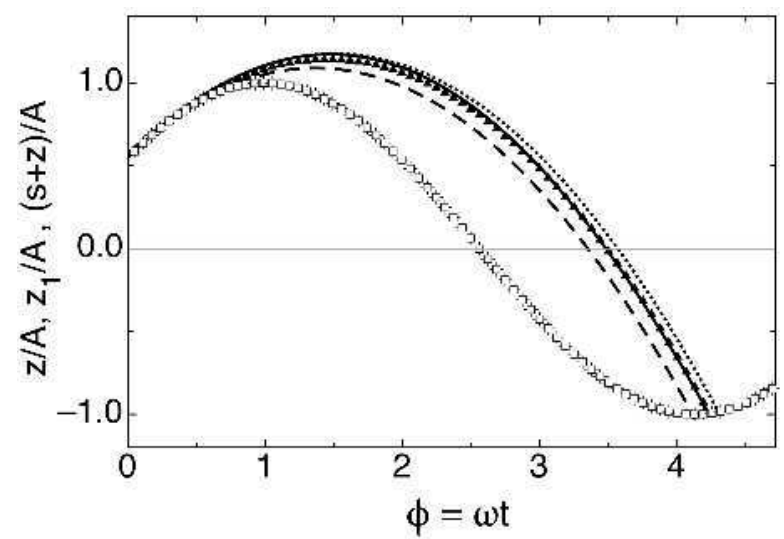

FIG. 4. Trajectory of the column bottom layer: Dimensionless free fall motion model, $z_{1} / A$, for different time delays (dotted line: $\delta t=1 \mathrm{~ms}$, small $\boldsymbol{\Delta}: \delta t=3 \mathrm{~ms}$, dashed line: $\delta t=5 \mathrm{~ms}$ ) and Eq. (1) trajectory estimation, $s+z$ (solid black line). Open crossed squares: $z / A$. $[\Gamma=1.81$ and $f=15 \mathrm{~Hz}]$.

tial conditions. Taking $t_{1}$ as the origin of the free fall of the lower layer we can assume that the initial position and velocity are those of the substrate at time $t_{1}$, i.e. $z_{1}=A \sin \left(\omega t_{1}\right)+A \omega \cos \left(\omega t_{1}\right)\left(t-t_{1}\right)-\frac{1}{2} g\left(t-t_{1}\right)^{2}$. Doing so, we expect a linear increase of $h$ with the velocity:

$$
\frac{d h}{d t}=\frac{1}{2} A \sqrt{1-\frac{1}{\Gamma^{2}}} \omega^{2} \delta t^{2}
$$

where we define $\delta t=t_{1}-t_{0}$, the delay between the origins of the free falls of the lower and upper surfaces. From the experimental slope, we get $\delta t=(4.7 \pm 0.2) \mathrm{ms}$.

It is then particularly interesting to discuss the physical origin of the delay. We already observed that the grains of the lower surface experience a free fall. One must however notice that the grains can be in free fall only if their motion is not frustrated. Note that, when they take off, their position and velocity are limited by the solid surface below and the grains above. Their velocity is oriented upwards and their acceleration equals the acceleration due to gravity only if their trajectory does not intersect

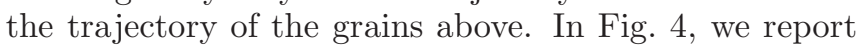
the trajectory of the bottom layer, $z_{1}(t)$, in free fall for several values of $\delta t$ (taking $t_{1}=t_{0}+\delta t$ ), and the altitude, $z(t)+s(t)$, estimated from the solution of Eq. (11) (black line in Fig. (4). We observe that for small $\delta t, z_{1}>z+s$, which means that the motion of the grains of the bottom surface is limited by the motion of the grains above ( $\delta t=$ $1 \mathrm{~ms}$, dotted line in Fig. (4). On the contrary, for large enough $\delta t, z_{1}<z+s$ at all time until the collision with the substrate. The grains can experience a free fall $(\delta t=$ $5 \mathrm{~ms}$, dashed line in Fig. (4). For intermediate values of $\delta t$, the trajectories, $z_{1}$ and $s+z$, cross each other at a time which compares with the collision time $(\delta t=3 \mathrm{~ms}$, small triangles in Fig. (4). The grains can experience a trajectory very similar to a free fall until it collides with 
substrate. From the latter simple observation, one can 486 deduce that a delay of, at least, $3 \mathrm{~ms}$ is necessary for the ${ }_{487}$ grains of the lower surface to fall freely and that $5 \mathrm{~ms}$ is 488 clearly an overestimate of $\delta t$.

Thus, the simple argument above gives a reasonable 490 range, 3 to $5 \mathrm{~ms}$, for the experimental delay $\delta t=4.7 \mathrm{~ms}, 49$ which validates the potential mechanism proposed to ac-492 count for the dilation. In summary, the grains of the 493 two free surfaces of the column experience free falls, the 494 take-off of the lower grains being delayed by the presence 495 of the dense column above which experience a trajec-496 tory governed by the interplay between the acceleration of gravity and the friction with the gaseous phase.

In conclusion, we observed the bouncing of a porous499 column of grains and measured the resulting variations00 of the pressure underneath. When interaction between the column and the gas are weak, because of the size and weight of the grains, the pressure is reasonably accounted for by considering the column as a porous solid, thus neglecting the column dilation. The latter is satisfactorily explained by considering that the grains of the upper and lower surfaces experience a free falls. In this framework, the dilation only results from a delay between the departure times and not from any pressure profile within the column that would repel the grains from one another.

\section{ACKNOWLEDGMENTS}

The authors acknowledge the financial support from the contracts ANR-09-BLAN-0389-01/Conicyt-011 and CNRS-Conicyt-PCCI12016.
[1] Q. Wu, Y. Andreopoulos, and S. Weinbaum, Physical ${ }_{525}$ Review Letters 93, 19, 194501, (2004).

[2] See for instance, F. J. Muzzio, T. Shinbrot and B. J.527 Glasser, Powder Technology 124, 1, (2002).

[3] R. A. Bagnold, The Physics of Blown Sand and Desert529 Dunes. (Methuen, London, 1954).

[4] B. Andreotti, L. Bonneau, and E. Clément, Geophys.531 Res. Lett., 35, L08306, (2008).

[5] S. T. Thoroddsen and A. Q. Shen, Phys. Fluids, 13, 4,533 (2001).

6] D. Lohse, R. Rauhe, R. Bergmann and D. Van Der Meer, 535 Nature, 432, 689, (2004).

[7] S. Deboeuf, P. Gondret and M. Rabaud, Phys. Rev. E, 79, 041306, (2009).

[8] C. Laroche, S. Douady and S. Fauve, J. Phys. (Paris), 539 50, 699, (1989).

540

[9] P. Evesque and J. Rajchenbach, Physical Review Letters541 62, 44, (1989).

[10] L.I. Reyes, I. Sánchez, G. Gutiérrez, Physica A. 358, 466,543 (2005).

[11] H. K. Pak and R. P. Behringer, Physical Review Letters545 71, 1832, (1993).

[12] H. K. Pak, E. Van Doorn, and R. P. Behringer, Physical 547 Review Letters 74, 4643, (1995).
[13] J. Duran, Physical Review Letters 87, 254301, (2001).

[14] L. Caballero and F. Melo, Physical Review Letters 93, 258001, (2004).

[15] J. C. Pastenes, J.-C. Géminard and F. Melo, Phys. Rev. E 88, 012201 (2013).

[16] W. Kroll, Forsch. auf der Geb. des Ing. 20, 2, (1854).

[17] R. G. Gutman, Trans. Instn. Chem. Engrs, 54, 174-183, (1976).

[18] See for instance, R.M. Nedderman, Statics and Kinematics of Granular Materials, (Cambridge Univ. Press 1992).

[19] Compressibility effect can be neglected if $\tau_{D} / \tau_{S}<<1$, where $\tau_{S}$ is the time during which the column sits on the vibrating surface and $\tau_{D}=\langle h\rangle^{2} / D$, with $D \equiv \kappa / \psi \eta \chi$ the relevant diffusion coefficient and $\chi$ the gas compressibility. Note that $\tau_{S}$ is a decreasing function of both $f$ and $\Gamma$. In our experimental conditions, for values of $\Gamma \lesssim 3$, compressibility effects become significant for $\Gamma \gtrsim 100 \stackrel{\mathrm{Hz}}{ }$.

[20] Notice that this result remains accurate for values of $\Gamma \lesssim 3$. Above this value, the flight time begins to deviate from the prediction of the inelastic ball model. Independent work indicates that this effect develops if the sitting time of the column on the vibrating plate becomes of the order of the collision time 21].

[21] J.M. Pastor, D. Maza, I. Zuriguel, A. Garcimartín and J.-F. Boudet, Physica D, 232, 128135, (2007). 